\title{
Membrane dipole potential of interaction between amyloid protein and phospholipid membranes is dependent on protein aggregation state
}

\author{
Steven S.-S. Wang *, Kuan-Nan Liu \\ Department of Chemical Engineering, National Taiwan University, Taipei 10617, Taiwan \\ Received 20 November 2007; received in revised form 2 March 2008; accepted 3 March 2008
}

\begin{abstract}
At least 20 different human proteins can fold abnormally resulting in the formation of pathological aggregates and several deadly degenerative diseases. Evidence also suggests that non-disease-associated proteins, under appropriate conditions, can aggregate in vitro to form amyloid fibrillar species. Numerous reports have shown that the interaction between cell membrane and amyloid proteins is of particular importance in the cytotoxic effects elicited by amyloid proteins. Despite the significant progress has been made, there are still large gaps in our knowledge of the disease mechanism(s) associated with this aforementioned interaction. In the current research, using a dual-wavelength fluorescence ratiometric method along with a voltage-sensitive dye, di-8-ANEPPS, we found that a decrease in intramembrane dipole potential was observed upon binding of amyloid proteins with phospholipids and this decrease became more dramatic when protein was in its aggregated form. Moreover, our data revealed that a correlation among the presence of cholesterol, the type of phospholipid, and the drop in dipole potential was evident. In comparison with the pure DPPC, the relative difference in dipole potential between fibrillar and freshly prepared samples attenuated with the addition of cholesterol while an increase in relative potential difference was observed in DPPG. Importantly, our results, for the first time, presented that the membrane dipole potential in amyloid protein-phospholipid interaction was dependent on the aggregation state of proteins, which is highly associated with the biological effects elicited by amyloid proteins.
\end{abstract}

(C) 2008 Taiwan Institute of Chemical Engineers. Published by Elsevier B.V. All rights reserved.

Keywords: Amyloid fibril; Aggregation state; Dipole potential; Phospholipid; Lysozyme; Lactalbumin

\section{Introduction}

The amyloidoses are complex, multiform disorders characterized by the assembly of normally innocuous and soluble proteins into extracellular insoluble fibrils. More than 20 different proteins so far have been identified as the fibrillar components of disease-associated amyloid deposits (Dobson, 2004; Ross and Poirier, 2004; Uversky and Fink, 2004; Wang and Good, 2005). While these diseases-associated amyloidogenic proteins demonstrate little sequence or structural homology, they all produce similar, straight, unbranched amyloid fibrils with characteristic cross- $\beta$-sheet structure and the ability to bind Congo red dye (Glenner, 1980; Kelly, 1996; Lansbury, 1999). Evidence suggests that non-disease related proteins can also be induced in vitro to polymerize into amyloid fibrils under certain conditions (Ferrao-Gonzales et al., 2000; Lai et al., 1996; Shtilerman et al., 2002; Sluzky et al., 1991, 1992). Several

\footnotetext{
* Corresponding author: Tel.: +88623366 5870; fax: +886223623040.

E-mail address: sswang@ntu.edu.tw (S.S.-S. Wang).
}

studies have shown that the type of solvent, $\mathrm{pH}$, chaperones, and protein concentration all affect peptide/protein structure and amyloid-formation in vitro (Castano and Frangione, 1995; Hilbich et al., 1991; Mantyh et al., 1993; Soto et al., 1995). Notably, the underlying mechanism by which amyloid fibrils form in vivo has yet to be completely determined.

A number of researchers have concluded from their observations and put forth several hypotheses accounting for the mechanism that triggers the interaction between amyloid proteins and cell membrane which has been regarded to be of paramount significance in the process of aggregation and amyloid fibrillogenesis (Arispe, 2004; Blanchard et al., 2002; Eliezer, 2006; Kagan et al., 2004; Kilsdonk et al., 1995; Neufeld et al., 1996; Quist et al., 2005; Varadarajan et al., 2000; Whitson et al., 1994). Furthermore, it has been indicated that the cell membrane is essential to the cytotoxic effects elicited by amyloid-forming proteins (Folin et al., 2006; Gharibyan et al., 2007; Shaked et al., 2006; Wood et al., 2003). Nevertheless, the mode and nature of the aforementioned interaction(s) of amyloid proteins with membranes still remain far from complete. 
Recently, numerous lines of evidence have indicated that the intramembrane molecular dipole is involved in the interactions of macromolecules and membranes (Asawakarn et al., 2001; Cladera and O'Shea, 1998; Cladera et al., 2003; StarkePeterkovic et al., 2006). Based on these findings, we hypothesized that the membrane dipole potential served as an influential parameter in the amyloid protein-membrane interaction. To that end, with hen egg-white lysozyme (HEWL) and bovine $\alpha$-lactalbumin used as model amyloid protein systems, we have utilized the dual-wavelength fluorescence ratiometric method combined with a voltage-sensitive dye, di-8-ANEPPS, to probe for the dipole potential upon interaction of model membranes with amyloid-forming proteins. We found that the magnitude of fluorescence ratio was affected by the aggregation state of protein, the type of phospholipid, and the presence of cholesterol in phospholipid. Importantly, our results, for the first time, demonstrated a close association between the membrane dipole potential of amyloid protein-membrane interaction and the aggregation state of proteins, which is highly associated with the biological effects elicited by amyloid proteins.

\section{Materials and methods}

\subsection{Materials}

Hen egg-white lysozyme was obtained from Merck (Germany). Bovine $\alpha$-lactalbumin ( $\alpha$-LA) was purchased from Sigma (USA). DPPC and DPPG vesicles were purchased from Avanti Polar Lipids (USA). Fluorescent dye 1-(3-sulfonatopropyl)-4-[ $\beta[2-($ di- $n$-octyl-amino)-6-naphthyl]vinyl]pyridinium betaine (di-8-ANEPPS) was obtained from Molecular Probes (USA). All other chemicals, unless otherwise specified, were obtained from Sigma (USA).

\subsection{Protein sample preparation}

Saline solution (136.7 mM NaCl, $2.68 \mathrm{mM} \mathrm{KCl,} \mathrm{pH} \mathrm{2.0)}$ with $0.01 \%(\mathrm{w} / \mathrm{v})$ sodium azide was used to prepare HEWL sample at $2 \mathrm{mg} / \mathrm{mL}$ or $\alpha$-LA sample at $1 \mathrm{mg} / \mathrm{mL}$. Protein sample solutions were first mixed via vortexing and then incubated in a reciprocating shaker incubator with $50 \mathrm{rpm}$ at $55^{\circ} \mathrm{C}$ during the course of experiment.

\subsection{Lipid vesicles preparation}

Phospholipid vesicles were prepared by dissolving DPPC or DPPG (with or without cholesterol) in chloroform/methanol $(2: 1, \mathrm{v} / \mathrm{v})$ solution and then the solvent was evaporated off under nitrogen. Sterile PBS was then added to suspend the lipid films of phospholipid, producing a lipid solution with concentration of $5.17 \mathrm{mM}$. The resulting suspensions were first sonicated for $20 \mathrm{~min}$ to obtain multilamellar vesicles (MLV). Next, the MLVs were frozen and thawed five times to obtain a more homogeneous distribution of large unilamellar vesicles (LUV). Finally, the LUVs were ultra-sonicated for $30 \mathrm{~min}$ to produce small unilamellar vesicles (SUV), and then extruded five times through filters of pore size $200 \mathrm{~nm}$ to remove the aggregates in the suspensions. The quality of the lipid vesicles was checked through the particle size distribution obtained from dynamic light scattering. The phospholipid vesicles were diluted to a final concentration of $2.07 \mathrm{mM}$ (2.5 times dilution of the initial phospholipid concentration) in sterile PBS prior to use.

\subsection{Dye labeling and dipole potential measurement}

The procedure of di-8-ANEPPS labeling was modified from ones used in the previous studies (Asawakarn et al., 2001; Montana et al., 1989; Vitha and Clarke, 2007). $2000 \mu \mathrm{L}$ of DPPC or DPPG $(2.07 \mathrm{mM})$ in sterile PBS with or without $30 \mathrm{wt} \%$ cholesterol were mixed with $1000 \mu \mathrm{L}$ protein, phloretin $(3 \mathrm{mM})$, or 6-ketocholestanol $(3 \mathrm{mM})$ and then incubated for at least $30 \mathrm{~min} .10 \mu \mathrm{L}$ of di-8-ANEPPS $(400 \mu \mathrm{M})$ were added into phospholipid-protein, phospholipid-phloretin, or phospholipidketocholestanol solutions and fluorescence spectra were taken at different incubation times. The ratio of fluorescence intensities excited at the two wavelengths of 460 and $520 \mathrm{~nm}$ at constant emission wavelength of $580 \mathrm{~nm}$ on an F-2500 Fluorescence Spectrophotometer (Hitachi, Japan) was used as a measure of membrane dipole potential.

\subsection{Thioflavin T fluorescence assay}

$40 \mu \mathrm{L}$ of lysozyme samples taken at different times were mixed with $960 \mu \mathrm{L}$ of $10 \mu \mathrm{M}$ thioflavin $\mathrm{T}$ (ThT) in phosphate buffered saline (PBS) with $0.01 \%(\mathrm{w} / \mathrm{v})$ sodium azide. ThT fluorescence intensity measurements were performed by exciting samples at $440 \mathrm{~nm}$ and recording emission intensities at $485 \mathrm{~nm}$ using an F-2500 Fluorescence Spectrophotometer (Hitachi, Japan). All measurements were taken in triplicate.

\subsection{Congo red binding assay}

To assess the presence of amyloid fibrils in sample solutions, Congo red binding studies were performed. Congo red dye was dissolved in sterile PBS to a final concentration of $112 \mu \mathrm{M}$. Congo red absorbances of protein sample solutions and the free dye controls were determined by adding Congo red to a final concentration of $18 \mu \mathrm{M}$ and acquiring spectral measurements from 400 to $700 \mathrm{~nm}$ at $25^{\circ} \mathrm{C}$ using a Spectronic Genesys 5 Spectrophotometer (Spectronic Instrument, USA) (Klunk et al., 1989). Both the protein solutions and the control solutions were allowed to interact with Congo red for at least $30 \mathrm{~min}$ prior to recording their spectra.

\subsection{Circular dichroism spectroscopy}

Circular dichroism (CD) spectra of protein samples were recorded on a JASCO J-810 (150-S Type) spectrometer (Sunway Scientific Corporation) at $25{ }^{\circ} \mathrm{C}$ using a bandwidth of $2.0 \mathrm{~nm}$ with step interval of $0.1 \mathrm{~nm}$, and an averaging time of $2 \mathrm{~s}$. A $0.2 \mathrm{~cm}$ quartz cell was used for far-UV (190-260 nm) measurements. Three scans each of duplicate samples were measured and averaged. Control buffer scans were run in 
duplicate, averaged, and then subtracted from the sample spectra. The results were plotted as ellipticity (millidegree) vs. wavelength $(\mathrm{nm})$. The secondary structure contents $(\alpha$-helix, $\beta$ sheet, and random coil) of the protein samples were estimated from the $\mathrm{CD}$ spectra using the software provided by the DICROWEB website (Whitmore and Wallace, 2004).

\subsection{Transmission electron microscopy}

A $20 \mu \mathrm{L}$ protein sample was mixed for $5 \mathrm{~min}$ in $0.25 \%$ glutaraldehyde (Electron Microscopy Sciences, Hatfield, PA, USA) in PBS, pH 7.2. The samples was then placed on carbon stabilized, formvar coated grids. Grids were negatively stained with 2\% (w/v) aqueous uranyl acetate (Electron Microscopy Sciences, Hatfield, PA, USA) and then examined and photographed in a JEOL, JEM-1200EXII transmission electron microscope (Tokyo, Japan) at an accelerating voltage of $80 \mathrm{kV}$.

\section{Results}

\subsection{Amyloid fibril formation as measured via spectroscopic methods}

At the outset, we needed to confirm that the freshly prepared and fibrillar samples had the expected structures. ThT fluorescenc spectroscopy and Congo red absorbance spectroscopy were employed to probe for the presence of cross- $\beta$ pleated sheet structures associated with amyloids (LeVine, 1993). An enhancement in ThT fluorescence emission has been regarded as an important indicator of the presence of amyloid fibril. Also, protein solutions containing amyloid fibrils shift the spectral properties of Congo red and exhibit a considerable increase in absorbance at around $540 \mathrm{~nm}$ (Klunk et al., 1989). As depicted in Fig. 1(a), the nucleation-based polymerization kinetics with sigmoidal time-course response was observed in the fibrillogenesis processes of HEWL and $\alpha$-LA. No perceptible increase in ThT-induced fluorescence was observed in the freshly prepared samples of HEWL and $\alpha$-LA. However, their fibrillar counterparts, HEWL at day 8 of incubation and $\alpha$ LA at day 2 of incubation, had considerable amyloid contents as ascertained by the increase in ThT fluorescence relative to the solvent control levels. Similar trend was also observed from the Congo red binding data (see Fig. 1(b)).

\subsection{Fibril morphology as detected via electron microscopy (EM)}

Confirmation of the protein ultrastructure formed during the aggregation or fibrillization process was evidenced by transmission electron microscopy. No fibrils were found in the freshly prepared samples of HEWL and $\alpha$-LA (micrographs not shown), while the presence of amyloid fibrils in the fibrillar counterparts was detected in representative electron micrographs shown in Fig. 2. While minor variations in the appearance existed between the fibrillar/aggregated species from these two different kinds of proteins, it was evident upon closer examination on EM micrographs that the preparations of both protein systems possessed
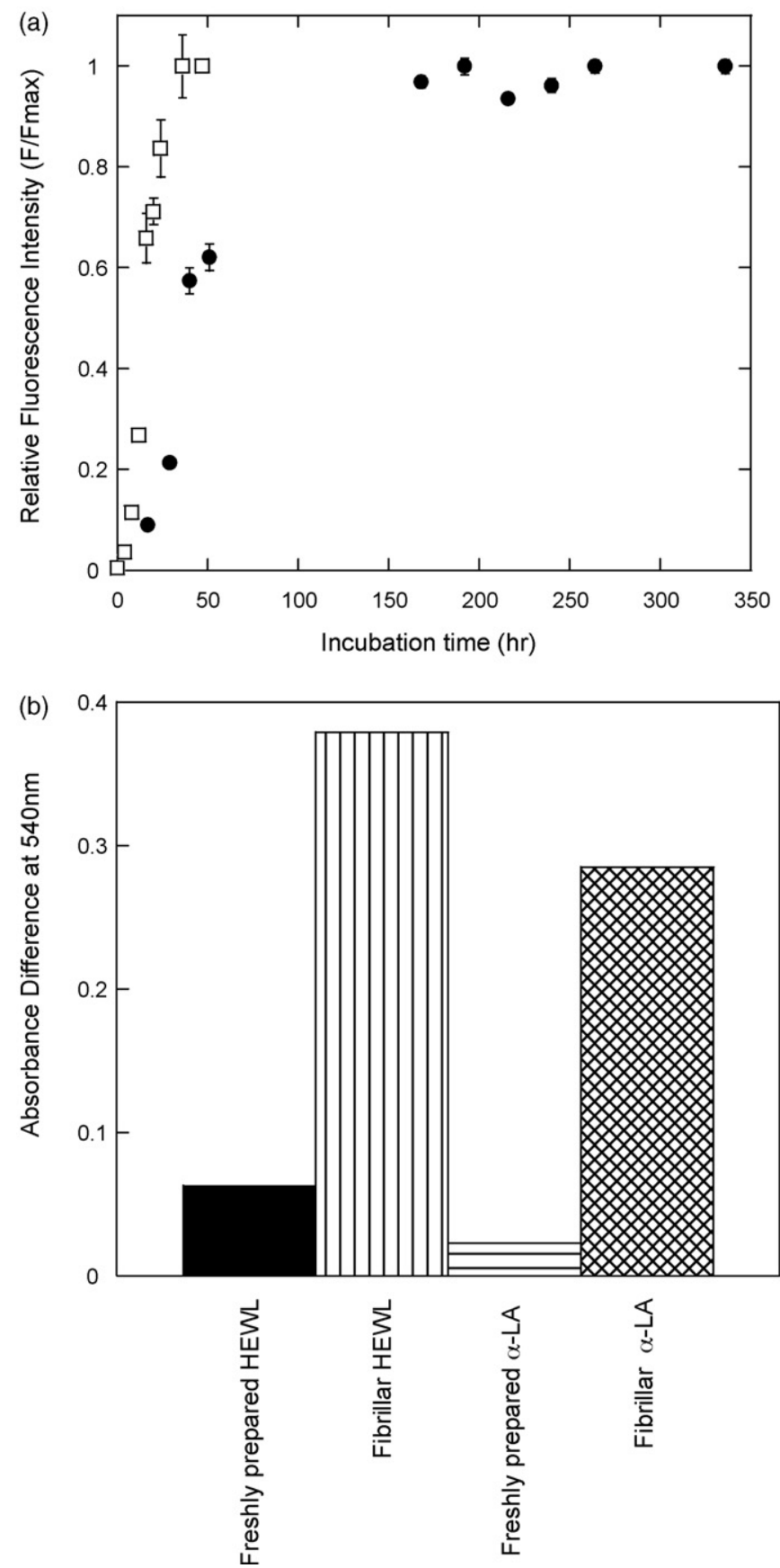

Fig. 1. The kinetics of aggregation or fibril formation of amyloid-forming proteins, hen egg-white lysozyme and bovine $\alpha$-lactalbumin. (a) The extent of fibril formation was measured via relative ThT fluorescence as a function of elapsed time (closed circle: hen egg-white lysozyme; open square: bovine $\alpha$ lactalbumin). (b) The extent of fibril formation was estimated via Congo red difference spectrum at wavelength of $540 \mathrm{~nm}$. The preparation of protein samples (HEWL: hen egg-white lysozyme; alpha-LA: $\alpha$-lactalbumin) was indicated in Section 2. The concentration of phospholipid (DPPC or DPPG) was $2.07 \mathrm{mM}$ and the concentrations of hen egg-white lysozyme and bovine $\alpha$ lactalbumin were 140 and $70 \mu \mathrm{M}$, respectively. Data represent the mean value of at least 4 independent experiments $(n \geq 4)$.

filamentous species reminiscent of typical amyloid fibrils with $\sim 10 \mathrm{~nm}$ in diameter and $\sim \mu \mathrm{m}$ in length. In addition, a sheet-like structure with a larger cross-sectional area composed of fibril bundles was observed in the fibrillar HEWL samples (Fig. 2(b)). 

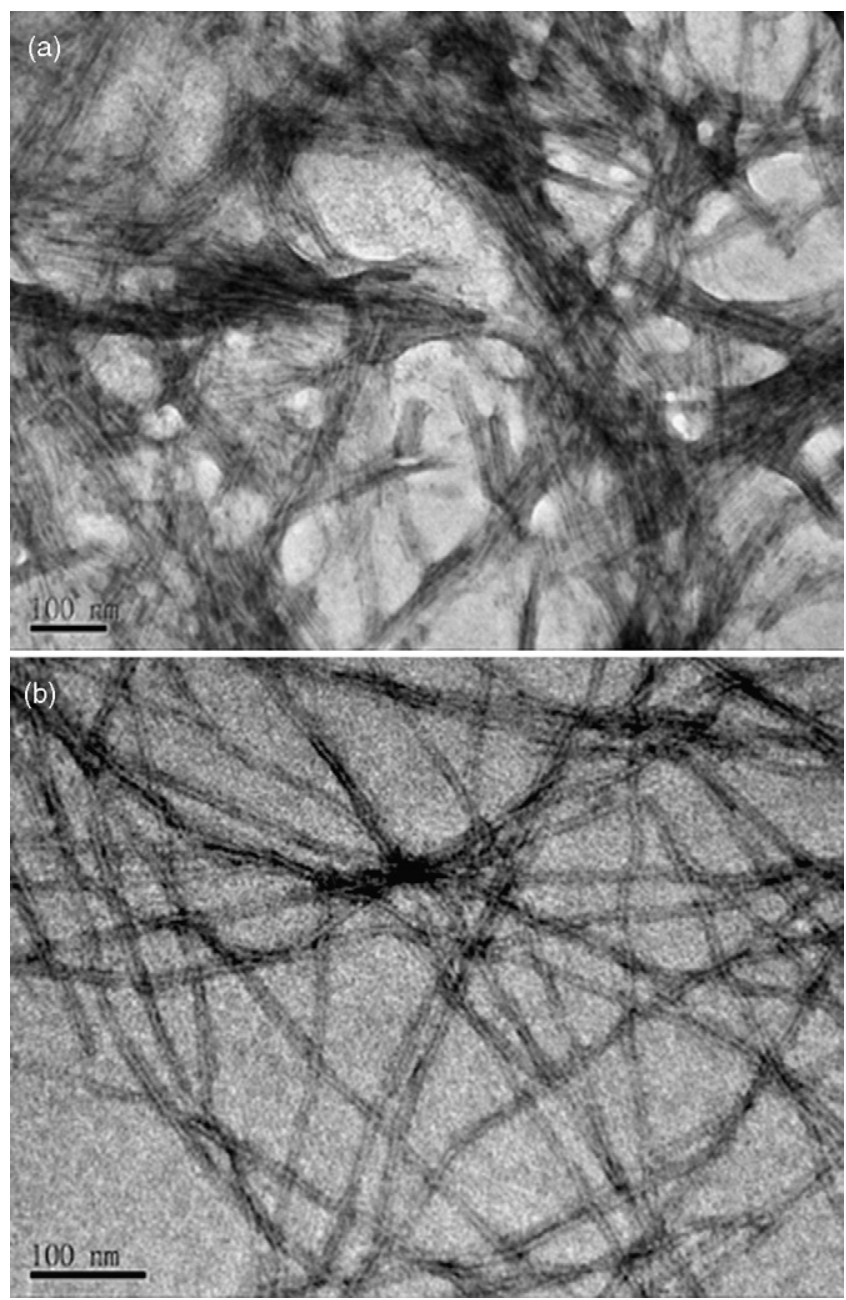

Fig. 2. Electron micrographs of negatively stained (a) hen egg-white lysozyme taken after 8 days of aggregation and (b) bovine $\alpha$-lactalbumin taken after 2 days of aggregation.

\subsection{Secondary structure as analyzed via circular dichroism}

The CD absorption spectroscopy was undertaken to characterize the secondary structure information of protein species formed during the amyloid fibrillization. As illustrated in Fig. 3(a) and (b), the far-UV CD spectra of freshly dissolved HEWL and $\alpha$-LA both displayed a shoulder at $\sim 222 \mathrm{~nm}$ and a signal minimum at $\sim 208 \mathrm{~nm}$, reflecting the presence of certain contents of $\alpha$-helix conformation in freshly dissolved protein samples. Nevertheless, the formation of fibrillar protein species (e.g., HEWL at 8 days of incubation, $\alpha$-LA at 2 days of incubation), by contrast, was manifested as substantial changes in the spectral shape of CD spectra in which a pronounced absorption minimum at $\sim 216-220 \mathrm{~nm}$ was observed, clearly demonstrating that the $\beta$-sheet content was significantly increased in the fibrillar protein samples. In addition, in order to retrieve better quantitative information regarding the structural transition, the $\mathrm{CD}$ spectra were further analyzed using the software provided by the DICROWEB website. Estimates of the secondary structural components of HEWL

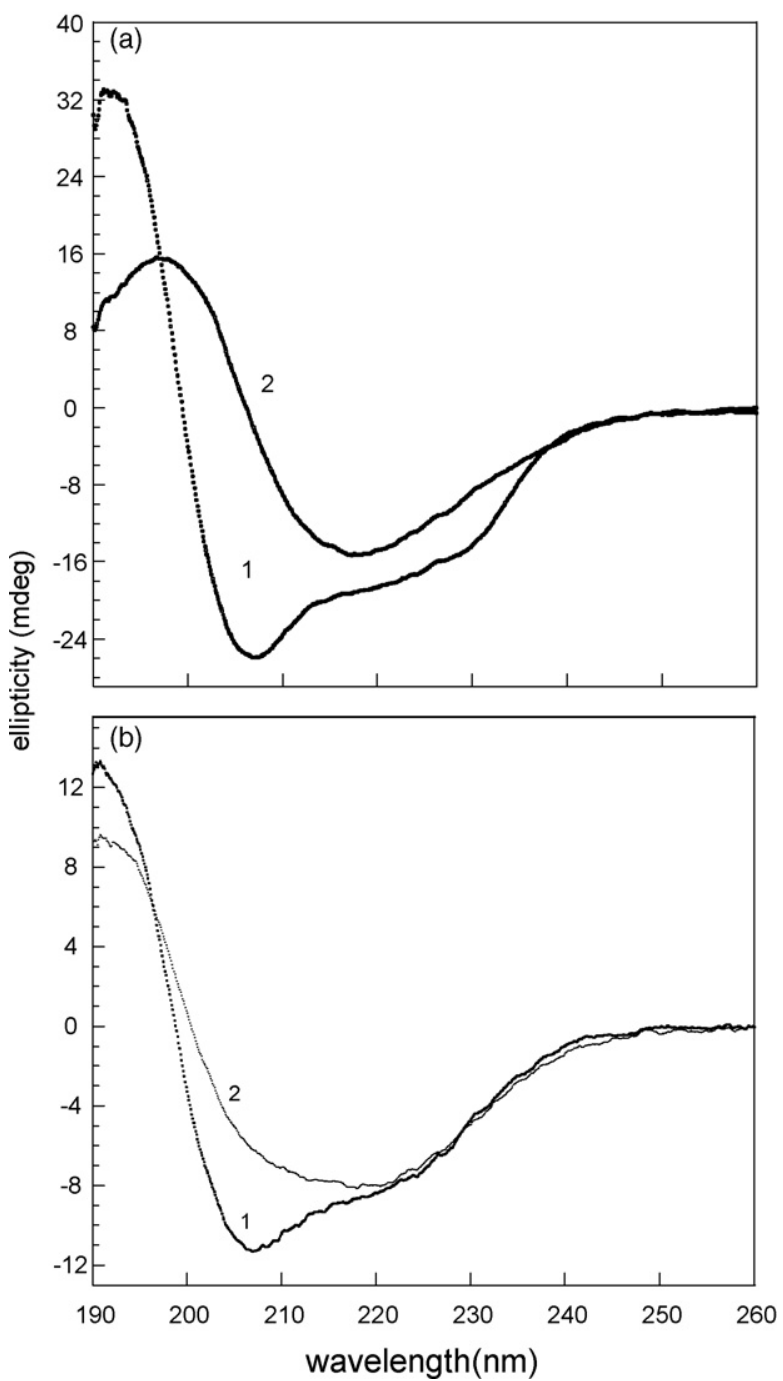

Fig. 3. (a) Representative CD spectra of hen egg-white lysozyme $(2 \mathrm{mg} / \mathrm{mL}$ or $140 \mu \mathrm{M}$ ) taken at two different incubation times (line 1:0 days of incubation (freshly prepared sample), line 2:8 days of incubation (fibrillar sample)). (b) Representative CD spectra of bovine $\alpha$-lactalbumin $(1 \mathrm{mg} / \mathrm{mL}$ or $70 \mu \mathrm{M})$ taken at two different incubation times (line 1:0 days of incubation (freshly prepared sample), line 2:2 days of incubation (fibrillar sample)).

samples are listed below: (1) freshly prepared HEWL: $33.2 \%$ helix, $12.7 \%$ strand, $14.3 \%$ turn, and $39.6 \%$ unordered; (2) fibrillar HEWL: $12.4 \%$ helix, $35.8 \%$ strand, $22.3 \%$ turn, and $29.5 \%$ unordered; (3) freshly prepared $\alpha$-LA: $24.8 \%$ helix, $18.5 \%$ strand, $16.6 \%$ turn, and $40.1 \%$ unordered; (4) fibrillar $\alpha$ LA: $18.2 \%$ helix, $25.4 \%$ strand, $18.2 \%$ turn, and $38.2 \%$ unordered.

\subsection{Optimization of dual-wavelength ratiometric fluorescence method}

It has been reported that variations in membrane dipole potential can be monitored using a dual-wavelength ratiometric fluorescence method along with a voltage-sensitive probe, di-8ANEPPS (Cladera and O'Shea, 1998; Cladera et al., 2001, 2003; Clarke, 2001; Gross et al., 1994). Upon changing the total membrane dipole moment, a shift in the fluorescence 
Table 1

Values of the fluorescence ratio, $R(460 / 520)$, and the percentage reduction in $R(460 / 520)$ for various protein-phospholipid systems

\begin{tabular}{|c|c|c|c|c|}
\hline & \multicolumn{2}{|l|}{ DPPC } & \multicolumn{2}{|l|}{ DPPG } \\
\hline & $R(460 / 520)$ & $\begin{array}{l}\text { Percentage reduction } \\
\text { in } R(460 / 520)(\%)\end{array}$ & $R(460 / 520)$ & $\begin{array}{l}\text { Percentage reduction } \\
\text { in } R(460 / 520)(\%)\end{array}$ \\
\hline 6-Ketocholestanol & 7.37 & NA & 9.26 & NA \\
\hline 6-Ketocholestanol (C) & 10.48 & NA & 11.50 & NA \\
\hline Phloretin & 1.16 & NA & 1.69 & NA \\
\hline Phloretin (C) & 5.03 & NA & 5.88 & NA \\
\hline Freshly prepared HEWL & 3.21 & 0.00 & 3.03 & 0.00 \\
\hline Fibrillar HEWL & 2.12 & 34.01 & 2.45 & 18.99 \\
\hline Freshly prepared HEWL (C) & 6.12 & 0.00 & 7.28 & 0.00 \\
\hline Fibrillar HEWL (C) & 5.02 & 17.94 & 5.17 & 29.01 \\
\hline Freshly prepared $\alpha$-LA & 3.08 & 0.00 & 3.41 & 0.00 \\
\hline Fibrillar $\alpha$-LA & 1.98 & 35.86 & 2.73 & 19.88 \\
\hline Freshly prepared $\alpha$-LA (C) & 10.1 & 0.00 & 8.85 & 0.00 \\
\hline Fibrillar $\alpha$-LA (C) & 7.27 & 28.31 & 5.62 & 36.48 \\
\hline $\mathrm{HCl}$ with salts & 3.59 & NA & 3.73 & NA \\
\hline $\mathrm{HCl}$ with salts (C) & 9.4 & NA & 7.69 & NA \\
\hline
\end{tabular}

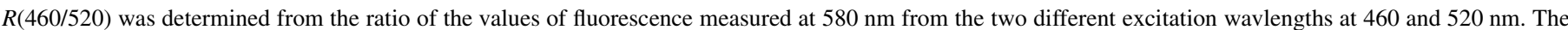

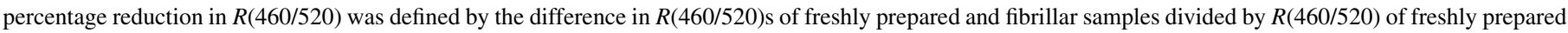

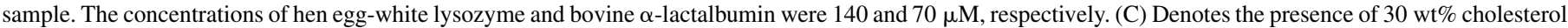
in phospholipid.

excitation spectrum of membrane-bound di-8-ANEPPS was observed. An index $R(460 / 520)$, determined by the ratio of fluorescence intensities at two excitation wavelengths, 460 and $520 \mathrm{~nm}$, at a fixed emission wavelength of $580 \mathrm{~nm}$, was taken as a convenient measure of relative change in the magnitude of membrane dipole potential (Cladera and O'Shea, 1998). The higher the $R(460 / 520)$ the more positive the dipole potential toward the center of lipid bilayer and vise versa. It has also been shown that the preparation of phospholipid membranes incorporated with certain sterols leads to an alteration in the membrane dipole. Phloretin, a sterol which was been shown to enhance the translocation rate of hydrophobic cations and decrease the rate of anions by dropping the positive dipole potential. Another compound, 6-ketocholestanol, has been found to exert the opposite effect on the membrane dipole (Cladera and O'Shea, 1998; Franklin and Cafiso, 1993; Simon et al., 1992). To achieve the optimal condition of dualwavelength ratiometric method, the effects of the two parameters used, the incubation time between protein sample and phospholipid membranes $\left(t_{1}\right)$ and the labeling time for di-8ANEPPS with protein-phospholipid mixture $\left(t_{2}\right)$, were first explored with the aid of these two sterols. Our results showed that no further changes in fluorescence signals were observed when $t_{1}$ and $t_{2}$ were greater than 2 and $13 \mathrm{~h}$, respectively. Therefore, these conditions were used for the following experiments.

\subsection{Relationship between protein aggregation state and membrane dipole potential induced by proteins- phospholipid interaction}

We were interested in knowing the effect of protein aggregation state on the change of intramembrane molecular dipoles induced by protein-membrane interaction. Employing the optimized dual-wavelength ratiometric procedure pre- viously described, we have investigated the influence of aggregation state (freshly prepared or fibrillar) of proteins on the membrane dipole potential of DPPC and DPPG, the neutral and negatively charged phospholipid vesicles, respectively. Table 1 shows the magnitudes of fluorescence ratios, $R(460 /$ $520)$, and the percentage reductions in $R(460 / 520)$ for various protein-phospholipid systems. As listed in Table 1, our results clearly demonstrated that a dramatic reduction in the fluorescence ratio $R(460 / 520)$ was observed when the fibrillar protein species were incubated with DPPC or DPPG vesicles in comparison with the vesicles in the buffer control $(\mathrm{HCl})$. On the contrary, the interaction of freshly prepared protein species with the phospholipids caused a decrease by lesser amount in the magnitude of $R(460 / 520)$. For instance, co-incubation of the fibrillar/aggregated HEWL or $\alpha$-LA with DPPC resulted in $R(460 / 520)$ that was lower than the freshly prepared counterpart by $\sim 34 \%$, or $\sim 36 \%$, respectively (see Table 1 ). However, in the case of DPPG, the value of $R(460 / 520)$ for the protein solution with fibrillar/aggregated species was approximately $80 \%$ of the freshly prepared counterpart (see Table 1). Overall, the interaction between aggregated/fibrillar species and phospholipid vesicles (DPPC or DPPG) led to a further decrease in dipole potential relative to freshly prepared species.

Similar experimental scheme was also applied to the phospholipid vesicles comprised of cholesterol. As apparently attested in Table 1, compared with the pure phospholipids, the presence of cholesterol in the membranes was responsible for the marked elevation in the magnitude of $R(460 / 520)$ in all conditions. Moreover, consistent with the results obtained in phospholipid vesicles (DPPC or DPPG) without cholesterol, exposure of cholesterol-containing phospholipid vesicles to the fibrillar/aggregated species led to a significant attenuation in the fluorescence ratios in comparison with their freshly prepared counterparts. We also discovered that, in HEWL, the percentage reduction in $R(460 / 520)$ ( $[R(460 / 520)$ of freshly 
prepared sample- $R(460 / 520)$ of fibrillar sample $] / R(460 / 520)$ of freshly prepared sample) relating to the interaction of fibrillar species with cholesterol-containing DPPG vesicles ( 29\%) was much smaller than that of DPPC without cholesterol ( $\sim 19 \%)$. However, an opposite trend was perceived for the interaction between the fibrillar species and DPPC vesicles (without cholesterol: $\sim 34 \%$; with cholesterol: $\sim 18 \%$ ) (see Table 1). Same conclusion could be made on $\alpha$-LA (as shown in Table 1).

\section{Discussion}

As of now, more than 20 different human proteins have been isolated as the fibrillar components of disease-associated amyloid deposits (Dobson, 2004; Ross and Poirier, 2004; Uversky and Fink, 2004; Wang and Good, 2005). Moreover, increasing evidence has revealed that proteins unrelated to any amyloidogenic disease can aggregate in vitro to form amyloid fibrillar species (Dobson, 2004; Fandrich et al., 2003; Kallberg et al., 2001).

A growing body of evidence has demonstrated that amyloid protein-membrane interactions have an impact on the cytotoxic effects elicited by amyloid proteins. These membraneassociated mechanisms of amyloid cytotoxicity include membrane depolarization (Blanchard et al., 2002; Whitson et al., 1994), membrane destabilization (Kilsdonk et al., 1995; Neufeld et al., 1996), pore or ion channel formation (Arispe, 2004; Eliezer, 2006; Kagan et al., 2004; Quist et al., 2005), and membrane-associated free radical generation (Butterfield et al., 1994; Butterfield, 1997; Varadarajan et al., 2000). Some researchers have demonstrated that amyloid proteins interact with membranes/phospholipids hydrophobically (Kremer et al., 2000) or electrostatically (Hertel et al., 1997; Seelig et al., 1995; Terzi et al., 1997). However, the results from the aforementioned studies have pointed out that, until now, the nature of the underlying mechanism of the amyloid proteinmembrane interaction is still under debate.

The membrane dipole potential exists between the highly hydrated lipid heads at the membrane surface and polar exterior of a membrane. The dipole potential arises from the orientation of aligned dipolar residues and water molecules in the vicinity of membrane surface (Cladera and O'Shea, 1998; Clarke, 2001; Klymchenko et al., 2003; Vitha and Clarke, 2007). It has been suggested that membrane dipole is involved in many biological processes, especially in the interactions of a variety of molecules (e.g., virus fusion peptides) with membranes, and could alter the folding and insertion of amphiphilic proteins (Cladera and O'Shea, 1998; Cladera et al., 1999, 2001; Rokitskaya et al., 1997; Zhang et al., 1996). We hypothesized that the dipole-dipole interaction is important in amyloid protein-membrane interaction and have set out to design experiments that examined the feasibility of this hypothesis.

In this study, we first prepared amyloid-forming proteins with two different aggregation states, namely freshly prepared and fibrillar/aggregated states, from two different proteins: hen lysozyme and bovine $\alpha$-lactalbumin. The formation of amyloid fibrils is particularly significant because a correlation appears to exist between the biological effects of amyloid-forming peptides and their aggregation states (Rymer and Good, 2001; Seilheimer et al., 1997; Ward et al., 2000). In contrast to the freshly prepared samples, the fibrillar protein samples were found to have formed amyloid fibrils under certain conditions through ThT fluorescence enhancement (Fig. 1(a)), Congo red binding assay (Fig. 1(b)), and transmission electron microscopy (Fig. 2). In addition, analogous to the results reported by the others (Ahmad et al., 2006; Pearce et al., 2007; Xu et al., 2007), our CD spectra indicated that the fibrillar protein samples possessed an essentially $\beta$-sheet rich conformation while either helix-rich conformation was detected in the freshly prepared counterparts (Fig. 3).

We then employed a dual-wavelength ratiometric method along with a voltage-sensitive fluorescent dye di-8-ANEPPS to monitor for changes in the membrane dipole potential during the interactions between phospholipid vesicles and amyloid proteins/peptides in the freshly prepared and fibrillar/aggregated state. Phospholipid vesicles, DPPC and DPPG, with or without cholesterol, were utilized as model membrane systems in this study. Cholesterol serves as an essential component of living cell membranes and is critical for normal cellular functions (Brown and Goldstein, 1997; Howland et al., 1998). Judging from the magnitudes of membrane dipole potentialassociated fluorescence ratio measured in our work (see Table 1), a number of points can be addressed as follows: (1) incubation of protein species with lipid vesicles (with or without cholesterol) resulted in a decrease in the membrane dipole potential, (2) the incorporation of cholesterol in the phospholipid vesicles gave a ratio that was higher than that of DPPC or DPPG alone, (3) in comparison with the pure phospholipids, exposure of the proteins to the membranes containing cholesterol, which assuredly more closely simulate the membranes systems in living cells, gave an opposite trend of the changes in membrane dipole potentials between freshly prepared and fibrillar protein samples, (4) a connection appeared to exist between the augmented aggregation/fibril formation and the decreased dipole potential, (5) compared with the pure neutral vesicle (DPPC), the relative difference in dipole potential between fibrillar and freshly prepared samples attenuated with the addition of cholesterol while an increase was observed in the negatively charged DPPG.

In conclusion, while further analysis is warranted regarding the details, the relationship between membrane dipole potential and amyloid protein-membrane interaction was discovered for the first time in this work. In addition, this relationship was found to be greatly dependent upon the aggregation state of proteins, which is closely associated with the biological effects induced by amyloid proteins. Collectively, our experimental observations strongly implicated the importance of membrane dipole potential in the amyloidforming protein-membrane interactions.

\section{Acknowledgement}

This work was supported by grants from the National Science Council, Taiwan. 


\section{References}

Ahmad, M. F., T. Ramakrishna, B. Raman, and C. M. Rao, "Fibrillogenic and Non-Fibrillogenic Ensembles of SDS-Bound Human Alpha-Synuclein," $J$. Mol. Biol., 364, 1061 (2006).

Arispe, N., "Architecture of the Alzheimer's A Beta P Ion Channel Pore," J. Membr. Biol., 197, 33 (2004).

Asawakarn, T., J. Cladera, and P. O'Shea, "Effects of the Membrane Dipole Potential on the Interaction of Saquinavir with Phospholipid Membranes and Plasma Membrane Receptors of Caco-2 Cells," J. Biol. Chem., 276, 38457 (2001).

Blanchard, B. J., V. L. Thomas, and V. M. Ingram, "Mechanism of Membrane Depolarization Caused by the Alzheimer Abeta1-42 Peptide," Biochem. Biophys. Res. Commun., 293, 1197 (2002).

Brown, M. S. and J. L. Goldstein, "The SREBP Pathway: Regulation of Cholesterol Metabolism by Proteolysis of a Membrane-Bound Transcription Factor," Cell, 89, 331 (1997).

Butterfield, D. A., "Beta-Amyloid-Associated Free Radical Oxidative Stress and Neurotoxicity: Implications for Alzheimer's Disease," Chem. Res. Toxicol., 10, 495 (1997).

Butterfield, D. A., K. Hensley, M. Harris, M. Mattson, and J. Carney, "BetaAmyloid Peptide Free Radical Fragments Initiate Synaptosomal Lipoperoxidation in a Sequence-Specific Fashion: Implications to Alzheimer's Disease," Biochem. Biophys. Res. Commun., 200, 710 (1994).

Castano, E. M. and B. Frangione, "Non-Alzheimer's Disease Amyloidoses of the Nervous System," Curr. Opin. Neurol., 8, 279 (1995).

Cladera, J. and P. O'Shea, "Intramembrane Molecular Dipoles Affect the Membrane Insertion and Folding of a Model Amphiphilic Peptide," Biophys. J., 74, 2434 (1998).

Cladera, J., I. Martin, J. M. Ruysschaert, and P. O'Shea, “Characterization of the Sequence of Interactions of the Fusion Domain of the Simian Immunodeficiency Virus with Membranes. Role of the Membrane Dipole Potential," J. Biol. Chem., 274, 29951 (1999).

Cladera, J., I. Martin, and P. O'Shea, “The Fusion Domain of HIV gp41 Interacts Specifically with Heparan Sulfate on the T-Lymphocyte Cell Surface," Embo J., 20, 19 (2001).

Cladera, J., P. O'Shea, J. Hadgraft, and C. Valenta, “Influence of Molecular Dipoles on Human Skin Permeability: Use of 6-Ketocholestanol to Enhance the Transdermal Delivery of Bacitracin," J. Pharm. Sci., 92, 1018 (2003).

Clarke, R. J., "The Dipole Potential of Phospholipid Membranes and Methods for Its Detection,” Adv. Colloid Interface Sci., 89, 263 (2001).

Dobson, C. M., "Principles of Protein Folding, Misfolding and Aggregation," Semin. Cell Dev. Biol., 15, 3 (2004).

Eliezer, D., "Amyloid Ion Channels: A Porous Argument or a Thin Excuse?" J. Gen. Physiol., 128, 631 (2006).

Fandrich, M., V. Forge, K. Buder, M. Kittler, C. M. Dobson, and S. Diekmann, "Myoglobin Forms Amyloid Fibrils by Association of Unfolded Polypeptide Segments," Proc. Natl. Acad. Sci. U.S.A., 100, 15463 (2003).

Ferrao-Gonzales, A. D., S. O. Souto, J. L. Silva, and D. Foguel, "The Preaggregated State of an Amyloidogenic Protein: Hydrostatic Pressure Converts Native Transthyretin into the Amyloidogenic State," Proc. Natl. Acad. Sci. U.S.A., 97, 6445 (2000).

Folin, M., S. Baiguera, D. Guidolin, R. Di Liddo, C. Grandi, E. De Carlo, G. G. Nussdorfer, and P. P. Parnigotto, "Apolipoprotein-E Modulates the Cytotoxic Effect of beta-Amyloid on Rat Brain Endothelium in an IsoformDependent Specific Manner," Int. J. Mol. Med., 17, 821 (2006).

Franklin, J. C. and D. S. Cafiso, "Internal Electrostatic Potentials in Bilayers: Measuring and Controlling Dipole Potentials in Lipid Vesicles," Biophys. J., 65, 289 (1993).

Gharibyan, A. L., V. Zamotin, K. Yanamandra, O. S. Moskaleva, B. A Margulis, I. A. Kostanyan, and L. A. Morozova-Roche, "Lysozyme Amyloid Oligomers and Fibrils Induce Cellular Death via Different Apoptotic/Necrotic Pathways," J. Mol. Biol., 365, 1337 (2007).

Glenner, G. G., "Amyloid Deposits and Amyloidosis: The Beta-Fibrilloses (Second of Two Parts)," N. Engl. J. Med., 302, 1333 (1980).

Gross, E., R. S. Bedlack, Jr., and L. M. Loew, "Dual-Wavelength Ratiometric Fluorescence Measurement of the Membrane Dipole Potential," Biophys. J., 67, 208 (1994).
Hertel, C., E. Terzi, N. Hauser, R. Jakob-Rotne, J. Seelig, and J. A. Kemp, "Inhibition of the Electrostatic Interaction between Beta-Amyloid Peptide and Membranes Prevents Beta-Amyloid-Induced Toxicity," Proc. Natl. Acad. Sci. U.S.A., 94, 9412 (1997).

Hilbich, C., B. Kisters-Woike, J. Reed, C. L. Masters, and K. Beyreuther, "Aggregation and Secondary Structure of Synthetic Amyloid Beta A4 Peptides of Alzheimer's Disease," J. Mol. Biol., 218, 149 (1991).

Howland, D. S., S. P. Trusko, M. J. Savage, A. G. Reaume, D. M. Lang, J. D. Hirsch, N. Maeda, R. Siman, B. D. Greenberg, R. W. Scott, and D. G. Flood, "Modulation of Secreted Beta-Amyloid Pprecursor Protein and Amyloid Beta-Peptide in Brain by Cholesterol," J. Biol. Chem., 273, 16576 (1998).

Kagan, B. L., R. Azimov, and R. Azimova, "Amyloid Peptide Channels," $J$. Membr. Biol., 202, 1 (2004).

Kallberg, Y., M. Gustafsson, B. Persson, J. Thyberg, and J. Johansson, "Prediction of Amyloid Fibril-Forming Proteins," J. Biol. Chem., 276, 12945 (2001).

Kelly, J. W., "Alternative Conformations of Amyloidogenic Proteins Govern Their Behavior," Curr. Opin. Struct. Biol., 6, 11 (1996).

Kilsdonk, E. P., P. G. Yancey, G. W. Stoudt, F. W. Bangerter, W. J. Johnson, M. C. Phillips, and G. H. Rothblat, "Cellular Cholesterol Efflux Mediated by Cyclodextrins," J. Biol. Chem., 270, 17250 (1995).

Klunk, W. E., J. W. Pettegrew, and D. J. Abraham, "Quantitative Evaluation of Congo Red Binding to Amyloid-like Proteins with a Beta-Pleated Sheet Conformation," J. Histochem. Cytochem., 37, 1273 (1989).

Klymchenko, A. S., G. Duportail, Y. Mely, and A. P. Demchenko, "Ultrasensitive Two-Color Fluorescence Probes for Dipole Potential in Phospholipid Membranes," Proc. Natl. Acad. Sci. U.S.A., 100, 11219 (2003).

Kremer, J. J., M. M. Pallitto, D. J. Sklansky, and R. M. Murphy, “Correlation of Beta-Amyloid Aggregate Size and Hydrophobicity with Decreased Bilayer Fluidity of Model Membranes," Biochemistry, 39, 10309 (2000).

Lai, Z., W. Colon, and J. W. Kelly, "The Acid-Mediated Denaturation Pathway of Transthyretin Yields a Conformational Intermediate that can SelfAssemble into Amyloid," Biochemistry, 35, 6470 (1996).

Lansbury, P. T., Jr. "Evolution of Amyloid: What Normal Protein Folding May Tell Us about Fibrillogenesis and Disease," Proc. Natl. Acad. Sci. U.S.A., 96, 3342 (1999).

LeVine, H., 3rd "Thioflavine T Interaction with Synthetic Alzheimer's Disease Beta-Amyloid Peptides: Detection of Amyloid Aggregation in Solution," Protein Sci., 2, 404 (1993).

Mantyh, P. W., J. R. Ghilardi, S. Rogers, E. Demaster, C. J. Allen, E. R. Stimson, and J. E. Maggio, "Aluminum, Iron, and Zinc Ions Promote Aggregation of Physiological Concentrations of Beta-Amyloid Peptide," J. Neurochem., 61, 1171 (1993).

Montana, V., D. L. Farkas, and L. M. Loew, "Dual-Wavelength Ratiometric Fluorescence Measurements of Membrane Potential," Biochemistry, 28, 4536 (1989)

Neufeld, E. B., A. M. Cooney, J. Pitha, E. A. Dawidowicz, N. K. Dwyer, P. G. Pentchev, and E. J. Blanchette-Mackie, "Intracellular Trafficking of Cholesterol Monitored with a Cyclodextrin," J. Biol. Chem., 271, 21604 (1996).

Pearce, F. G., S. H. Mackintosh, and J. A. Gerrard, "Formation of Amyloid-like Fibrils by Ovalbumin and Related Proteins under Conditions Relevant to Food Processing," J. Agric. Food Chem., 55, 318 (2007).

Quist, A., I. Doudevski, H. Lin, R. Azimova, D. Ng, B. Frangione, B. Kagan, Ghiso, and R. Lal, "Amyloid Ion Channels: A Common Structural Link for Protein-Misfolding Disease," Proc. Natl. Acad. Sci. U.S.A., 102, 10427 (2005).

Rokitskaya, T. I., Y. N. Antonenko, and E. A. Kotova, "Effect of the Dipole Potential of a Bilayer Lipid Membrane on Gramicidin Channel Dissociation Kinetics," Biophys. J., 73, 850 (1997).

Ross, C. A. and M. A. Poirier, "Protein Aggregation and Neurodegenerative Disease," Nat. Med., 10 Suppl., S10 (2004).

Rymer, D. L. and T. A. Good, "The Role of G Protein Activation in the Toxicity of Amyloidogenic Abeta-(1-40), Abeta-(25-35), and Bovine Calcitonin," J. Biol. Chem., 276, 2523 (2001).

Seelig, J., R. Lehrmann, and E. Terzi, "Domain Formation Induced by LipidIon and Lipid-Peptide Interactions," Mol. Membr. Biol., 12, 51 (1995). 
Seilheimer, B., B. Bohrmann, L. Bondolfi, F. Muller, D. Stuber, and H. Dobeli, "The Toxicity of the Alzheimer's Beta-Amyloid Peptide Correlates with a Distinct Fiber Morphology," J. Struct. Biol., 119, 59 (1997).

Shaked, G. M., M. P. Kummer, D. C. Lu, V. Galvan, D. E. Bredesen, and E. H. Koo, "A Beta Iinduces Cell Death by Direct Interaction with Its Cognate Extracellular Domain on APP (APP 597-624)," Faseb. J., 20, 1254 (2006).

Shtilerman, M. D., T. T. Ding, and P. T. Lansbury, "Molecular Crowding Accelerates Fibrillization of Alpha-Synuclein: Could an Increase in the Cytoplasmic Protein Concentration induce Parkinson's Disease?" Biochemistry, 41, 3855 (2002).

Simon, S. A., T. J. McIntosh, A. D. Magid, and D. Needham, "Modulation of the Interbilayer Hydration Pressure by the Addition of Dipoles at the Hydrocarbon/Water Interface," Biophys. J., 61, 786 (1992).

Sluzky, V., J. A. Tamada, A. M. Klibanov, and R. Langer, "Kinetics of Insulin Aggregation in Aqueous Solutions upon Agitation in the Presence of Hydrophobic Surfaces," Proc. Natl. Acad. Sci. U.S.A., 88, 9377 (1991).

Sluzky, V., A. M. Klibanov, and R. Langer, "Mechanism of Insulin Aggregation and Stabilization in Agitated Aqueous-Solutions," Biotechnol. Bioeng., 40, 895 (1992).

Soto, C., E. M. Castano, B. Frangione, and N. C. Inestrosa, "The Alpha-Helical to Beta-Strand Transition in the Amino-Terminal Fragment of the Amyloid BetaPeptide Modulates Amyloid Formation," J. Biol. Chem., 270, 3063 (1995).

Starke-Peterkovic, T., N. Turner, M. F. Vitha, M. P. Waller, D. E. Hibbs, and R. J. Clarke, "Cholesterol Effect on the Dipole Potential of Lipid Membranes," Biophys. J., 90, 4060 (2006).

Terzi, E., G. Holzemann, and J. Seelig, "Interaction of Alzheimer Beta-Amyloid Peptide(1-40) with Lipid Membranes," Biochemistry, 36, 14845 (1997).

Uversky, V. N. and A. L. Fink, "Conformational Constraints for Amyloid Fibrillation: The Importance of Being Unfolded," Biochim. Biophys. Acta, 1698, 131 (2004).
Varadarajan, S., S. Yatin, M. Aksenova, and D. A. Butterfield, "Review: Alzheimer's Amyloid Beta-Peptide-Associated Free Radical Oxidative Stress and Neurotoxicity,” J. Struct. Biol., 130, 184 (2000).

Vitha, M. F. and R. J. Clarke, "Comparison of Excitation and Emission Ratiometric Fluorescence Methods for Quantifying the Membrane Dipole Potential," Biochim. Biophys. Acta, 1768, 107 (2007).

Wang, S. S. S. and T. A. Good, "An Overview of Alzheimer's Disease," J. Chin. Inst. Chem. Engrs., 36, 533 (2005).

Ward, R. V., K. H. Jennings, R. Jepras, W. Neville, D. E. Owen, J. Hawkins, G. Christie, J. B. Davis, A. George, E. H. Karran, and D. R. Howlett, "Fractionation and Characterization of Oligomeric, Protofibrillar and Fibrillar Forms of Beta-Amyloid Peptide [In Process Citation]," Biochem. J., 348 (Pt 1), 137 (2000).

Whitmore, L. and B. A. Wallace, "DICHROWEB, an Online Server for Protein Secondary Structure Analyses from Circular Dichroism Spectroscopic Data," Nucleic Acids Res., 32, W668 (2004).

Whitson, J. S., M. P. Mims, W. J. Strittmatter, T. Yamaki, J. D. Morrisett, and S. H. Appel, "Attenuation of the Neurotoxic Effect of A Beta Amyloid Peptide by Apolipoprotein E,' Biochem. Biophys. Res. Commun., 199, 163 (1994).

Wood, W. G., G. P. Eckert, U. Igbavboa, and W. E. Muller, "Amyloid BetaProtein Interactions with Membranes and Cholesterol: Causes or Casualties of Alzheimer's Disease," BBA-Biomembranes, 1610, 281 (2003).

Xu, M., V. A. Shashilov, V. V. Ermolenkov, L. Fredriksen, D. Zagorevski, and I. K. Lednev, "The First Step of Hen Egg White Lysozyme Fibrillation, Irreversible Partial Unfolding, is a Two-State Transition," Protein Sci., 16, 815 (2007).

Zhang, J., L. M. Loew, and R. M. Davidson, "Faster Voltage-Dependent Activation of Na+ Channels in Growth Cones Versus Somata of Neuroblastoma N1E-115 Cells," Biophys. J., 71, 2501 (1996). 